\title{
Whole-brain connectivity during encoding: age-related differences and associations with cognitive and brain structural decline
}

Elettra Capogna ${ }^{a}$, Markus H. Sneve ${ }^{a}$, Liisa Raud ${ }^{a}$, Line Folvik ${ }^{a}$, Hedda Ness ${ }^{a}$, Kristine B. Walhovd ${ }^{a, b}$, Anders M. Fjella,b, Didac Vidal-Piñeiroa

${ }^{a}$ Centre for Lifespan Changes in Brain and Cognition, Department of Psychology, University of Oslo, Oslo, Norway.

${ }^{\mathrm{b}}$ Department of radiology and nuclear medicine, Oslo University Hospital, Oslo, Norway

Corresponding author:

\section{Elettra Capogna}

Department of Psychology, Pb. 1094, Blindern

Oslo, Norway, 0317

elettra.capogna@psykologi.uio.no

Tel: (+47) -22845061

Running title: Age-related differences in encoding connectivity 


\section{Abstract}

There is a limited understanding of age differences in functional connectivity during memory encoding. In the present study, a sample of cognitively healthy adult participants $(n=488)$, a subsample of whom had longitudinal cognitive and brain structural data spanning 8 years back, underwent $\mathrm{fMRI}$ while performing an associative memory encoding task. We investigated 1) age changes in whole-brain connectivity during memory encoding; whether 2) encoding connectivity patterns overlap with the activity signatures of specific cognitive processes and whether 3) connectivity changes associated with memory encoding related to longitudinal brain structural and cognitive changes. Age was associated with decreased intranetwork connectivity and increased connectivity during encoding. Task-connectivity between mediotemporal and posterior parietal regions - which overlapped with areas involved in mental imagery - was related to better memory performance only in older age. The connectivity patterns supporting memory performance in older age reflected preservation of thickness of the medial temporal cortex. These investigations collectively indicate that functional patterns of connectivity should be interpreted in accordance with a maintenance rather than a compensation account.

\section{Keywords}

Aging, functional connectivity, encoding, psychophysiological interaction (PPI), longitudinal MRI, episodic memory 


\section{Introduction}

Episodic memory declines with age (Rönnlund et al., 2005), although there is substantial interindividual variability in the trajectories (Nyberg et al., 2012). Variability in memory function is affected by changes in the structural and functional architecture of the brain. Hence, how brain regions communicate during memory tasks may be a key factor in explaining age-related changes in memory performance as well as inter-individual variation of performance in older age. Assessing brain functional connectivity changes in task contexts provides a window for studying age-related brain changes in response to specific cognitive demands (Campbell and Schacter, 2017). In the present study, we used task-related functional magnetic resonance imaging (fMRI) in a large adult lifespan sample to investigate whether 1) age is associated with changes in task-connectivity during encoding, specifically with decreased intranetwork and increased internetwork connectivity; 2) encoding connectivity patterns overlap with the activity signatures of specific cognitive processes; 3 ) connectivity changes associated with memory encoding performance relate to longitudinal brain structural and cognitive changes.

Most previous studies on functional connectivity changes in aging have employed seed-based taskfunctional connectivity (Grady et al., 2003; Oh and Jagust, 2013), or resting-state fMRI (rs-fMRI) (Fjell et al., 2015). Seed-based task-connectivity studies have repeatedly found higher age to be related to greater connectivity between medial temporal lobe, most notably the hippocampus, and prefrontal areas, during encoding (Grady et al., 2003; Oh and Jagust, 2013). Research using rs-fMRI has found lower intranetwork connectivity with higher age - especially within the default-mode regions - and increased connectivity between networks such as the dorsal attention and the defaultmode network (Sala-Llonch et al., 2015; Vidal-Piñeiro et al., 2014). However, encoding connectivity exhibits a substantially different pattern from that observed during rest (Keerativittayayut et al., 2018). Therefore, it is crucial to understand how whole-brain task-connectivity during memory encoding contributes to successful recollection. Encoding-based connectivity has been characterized by increased communication between distant areas, such as higher integration of 
default mode, salience, and subcortical networks with the other subnetworks (Keerativittayayut et al., 2018). Furthermore, flexible nodes - nodes that change networks membership during different episodic memory task phases - appear to be relevant for memory performance as degree of observed reorganization between states partially predicts retrieval success (Schedlbauer and Ekstrom, 2019). A small number of studies have tested age-related differences in whole-brain connectivity during memory encoding (Grady et al., 2016; Matthäus et al., 2012; Wang et al., 2010). Wang and colleagues (2010) found higher age to be associated with lower long-range functional connections of frontal regions with the rest of the brain during encoding. Matthaus et al. (2012) observed age-related increases in the density and size of the networks together with reduced efficiency of information processing during encoding.

Age-related functional changes may accompany brain structure decline (Nyberg et al., 2012). For instance, in accordance with the brain maintenance framework, older adults showing longitudinal changes in prefrontal activity - and in further areas beyond task-specific regions - exhibited greater memory and hippocampal volume decline (Persson et al., 2006; Pudas et al., 2018). Alternatively, age-differences in connectivity may reflect an attempt to compensate for neural breakdown (Cabeza et al., 2018). For instance, one study found that higher connectivity between the prefrontal cortex with the rest of the brain was related to sustained memory performance uniquely in older adults (Deng et al., 2021), supporting a compensatory account for the age-related connectivity changes. In general, coupling age-related differences in function with cross-sectional performance is however not without problems, as compensatory responses can lay anywhere along a continuum from (partial) failure to success (Grady, 2012). Hence, for a better and complete understanding, functional differences need to be associated with brain and cognitive changes assessed over time.

Here, we investigated age-related differences in functional connectivity during an associative encoding task using a correlational psychophysiological interaction (cPPI) approach (Fornito et al., 2012) and a sample encompassing the entire adult age range. We assessed connectivity changes during encoding associated with age, memory performance, and the interaction between age and 
memory. Specifically, we focused on whether age was associated with decreased intranetwork and increased internetwork connectivity during the task, as typically shown in whole-brain resting-state (Geerligs et al., 2015) and in ROI-based task-connectivity studies (Grady et al., 2016; Spreng et al., 2016). Moreover, by comparing connectivity maps with meta-analytic activity maps, we investigated whether encoding connectivity patterns overlapped with the activity signatures of specific cognitive processes. Finally, among older adults, we tested whether the connectivity changes associated with successful memory encoding were related to longitudinal structural and cognitive changes. This allowed us to test whether these functional patterns of connectivity should be interpreted in accordance with the maintenance or the compensation accounts.

\section{Material and methods}

\subsection{Participants}

A total of 488 participants (336 females, mean age $=41.65$ years, $S D=17.20$, age range $=18-81$ ) were included in the final sample. All participants completed the fMRI tasks and were screened through health and neuropsychological assessments. Participants were required to have no history of neurological or psychiatric disorders, chronic illness, be right-handed, and not to use medicines known to affect nervous system functioning. Participants were further excluded based on the following neuropsychological criteria: score $<26$ on the Mini-Mental State Examination (MMSE) (Folstein et al., 1975), score $<85$ on the WASI II (Wechsler, 1999), and a T-score of $\leq 30$ on the California Verbal Learning Test II_Alternative Version (CVLT II) (Delis et al., 2000) immediate delay and long delay. All participants gave written informed consent, and the study was approved by the Regional Ethical Committee of South Norway and conducted in accordance with the Helsinki declaration. Retrospective longitudinal data were available for a subsample of older participants (age $>50$ years), spanning up to 10 years back as follows: neuropsychological testing for 151 participants ( $\mathrm{n}=51,6$, and 94 with 1,2 , and $\geq 3$ observations, respectively), and brain structural scans for 88 
participants ( $n=2,5$, and 81 with 1,2 , and $\geq 3$ observations, respectively). Note that a small subsample of participants had retrospective data acquired with a different scanner. See Supplementary Table 1 for more information.

\subsection{Experimental design and behavioral analysis}

The experiment included an incidental encoding task and a memory test after approximately 90 minutes, both in the scanner. In this study, we only analyzed encoding fMRI data. The experimental design has been thoroughly described elsewhere (Sneve et al., 2015; Vidal-Piñeiro et al., 2019). See

Fig. 1 for a visual description of the experiment. In brief, the encoding and retrieval tasks consisted of 2 and 4 runs, respectively, that included 50 trials each. The stimulus material comprised 300 black and white line drawings of everyday items. A central fixation cross was shown during the baseline recording at the beginning, the middle, and the end of each run for 11 seconds. In the encoding phase, the trial started with a voice asking the participants either "Can you eat it?" or "Can you lift it?". Each question was asked 25 times in each run in a pseudorandomized order. One second after the question, an item appeared on the screen for 2 seconds, asking the participant to answer "Yes" or "No", before being replaced by a fixation cross that remained throughout the intertrial interval (between 1 and 7 seconds, exponential distribution; duration $=2.98$ [2.49] seconds). In the retrieval phase, the trial started with Question 1: "Have you seen this item before?". The item appeared on the screen for 2 seconds, and the participant had to press "Yes" (old item), or "No" (new item). In each run, 25 old items and 25 new items were presented in a pseudorandomized order. If the participant responded "No", the trial ended. If the participant responded "Yes", the trial proceeded to Question 2: "Can you remember what you were supposed to do with the item?". Again, if the participant responded "No", the trial ended, if "Yes" the trial continued with Question 3: "Were you supposed to eat it or lift it?". The participant was forced to choose between the two actions associated with the item at encoding. For behavioral analysis, the classification of responses to old items was: (1) source memory ("Yes" response to Question 1 and Question 2, and correct answer to Question 3), (2) item memory ("Yes" response to Question 1 and either "No" to Question 2 or incorrect answer to Question 3), (3) miss (incorrect answer to Question 1). New items were classified either as (4) 
correct rejections or (5) false alarms. Memory performance in the task was calculated as the proportion of source memory minus incorrect judgments to Question 3, tentatively controlling for false memories and guessing behavior (Vidal-Piñeiro et al., 2019). The relationship between age and relevant behavioral and neuropsychological metrics was tested with generalized additive models (GAMs), controlling for sex. 


\section{A) Encoding trials} 12

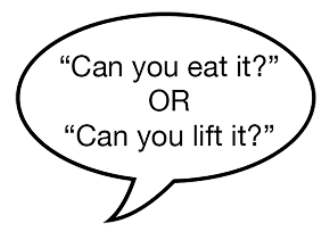

1s

$1 / 3 / 5 / 7 s$

$\sqcap \square$

B) Retrieval trials

\begin{tabular}{l|ll|l|l|l|l|l}
1 & 2 & 3 & 4
\end{tabular}

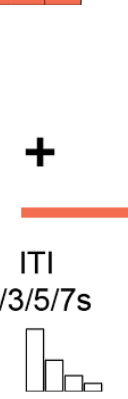

Q1

"Have you seen

this item before?"

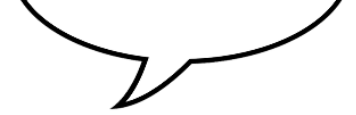

1s

Yes

No

Trial ends

ISI

$1 / 3 / 5$ s

$\Pi$

$2 s$

"Do you remember

what you were

asked to do with it?"

0

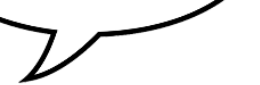

\begin{tabular}{|l|l|l}
\hline & $\square$ & $\square$
\end{tabular}

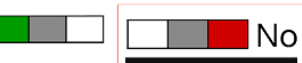

Trial ends

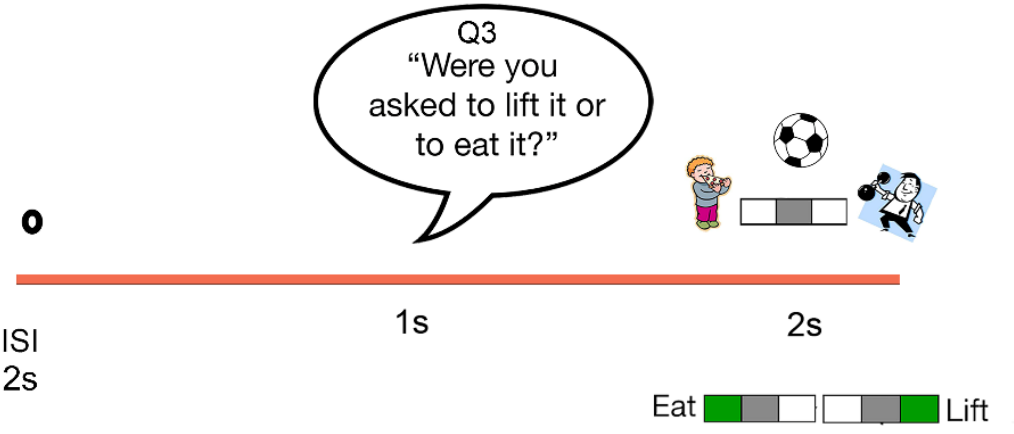


Fig.1 Experimental paradigm. A One trial of the encoding task. The green $\mathrm{V}$ and the red $\mathrm{X}$ were present on the screen to indicate which button indicate Yes and No. B One trial of the retrieval task. Test Questions 1 and 2 required a Yes/No response, whereas Question 3 required a choice between the two actions. The trial ended if the participant responded No to either one of the two first questions. Response cues ( $\mathrm{V}, \mathrm{X}$, eating, lifting) were also present on the screen. ITI intertrial interval, ISI interstimulus interval. Adapted from Vidal-Piñeiro and colleagues (2017).

\subsection{MRI acquisition}

Imaging data were collected using a 20-channel Siemens head-neck coil on a 3T MRI (Siemens Skyra Scanner, Siemens Medical Solutions, Germany) at Rikshospitalet, Oslo University Hospital. The functional imaging parameters were equivalent across all fMRI runs: 43 transversally oriented slices (no gap) were measured using a BOLD-sensitive T2*-weighted EPI sequence (TR = $2390 \mathrm{ms,}$ $\mathrm{TE}=30 \mathrm{~ms}$, flip angle $=90^{\circ}$, voxel size $=3 \times 3 \times 3 \mathrm{~mm}^{3}, \mathrm{FOV}=224 \times 224 \mathrm{~mm}^{2}$, interleaved acquisition; generalized autocalibrating partially parallel acquisitions acceleration factor [GRAPPA] $=2$ ). Each encoding run produced 134 volumes. At the start of each fMRI run, 3 dummy volumes were collected to avoid T1 saturation effects. Anatomical T1-weighted (T1w) magnetization-prepared rapid gradient echo (MPRAGE) images consisting of 176 sagittally oriented slices were obtained using a turbo field echo pulse sequence $\left(\mathrm{TR}=2300 \mathrm{~ms}, \mathrm{TE}=2.98 \mathrm{~ms}, \mathrm{TI}=850 \mathrm{~ms}\right.$, flip angle $=8^{\circ}$, voxel size $=1 \times 1 \times 1 \mathrm{~mm}^{3}, \mathrm{FOV}=256 \times 256 \mathrm{~mm}^{2}$ ) were also acquired. Furthermore, a standard double-echo gradient-echo field map sequence was acquired for distortion correction of the echo planar images. Visual stimuli were presented in the scanner environment with an NNL 32-inch LCD monitor while participants responded using the ResponseGrip device (both NordicNeuroLab, Norway). Auditory stimuli were presented to the participants' headphones through the scanner intercom. The structural T1w data used in the longitudinal analysis were collected using a 12-channel head coil on a $1.5 \mathrm{~T}$ Siemens Avanto scanner (Siemens Medical Solutions, Germany) at Rikshospitalet, Oslo University Hospital. The pulse sequence acquired consisted of two repeated 160-slice sagittal T1-weighted MPRAGE sequences $(T R=2400 \mathrm{~ms}, \mathrm{TE}=3.61 \mathrm{~ms}, \mathrm{TI}=1000 \mathrm{~ms}$, flip angle $=8^{\circ}$, voxel size $=1.25 \times 1.25 \times 1.20 \mathrm{~mm}$, FOV $=240 \mathrm{~mm}$ ). The raw images were automatically corrected for spatial distortion due to gradient nonlinearity (Jovicich et al., 2006) and field inhomogeneity (Sled et al., 1998), averaged, and resampled to isotropic $1 \mathrm{~mm}$ voxels. 


\subsection{MRI preprocessing}

\subsection{1 fMRI preprocessing}

Data were organized and named according to the Brain Imaging Dataset Specification standard (BIDS) and preprocessed using a fMRIPep preprocessing pipeline (Esteban et al., 2019) (v. 1.2.5) a "Nipype" based tool (Gorgolewski et al., 2018) (v. 1.1.6).

The T1w image was corrected for intensity non-uniformity (INU) using N4BiasFieldCorrection (Tustison et al., 2010) (ANTs v. 2.2.0), and used as T1w-reference throughout the workflow. The T1w-reference was then skull-stripped using antsBrainExtraction.sh (ANTs v. 2.2.0), using OASIS as target template. Brain surfaces were reconstructed using recon-all (FreeSurfer v. 6.0.1) (Dale et al., 1999), and the brain mask estimated previously was refined with a custom variation of the method to reconcile ANTs-derived and FreeSurfer-derived segmentation of the cortical gray matter (GM) of Mindboggle (Klein et al., 2017). Spatial normalization to the ICBM152 Nonlinear Asymmetrical template version 2009c (Fonov et al., 2009) was performed through nonlinear registration with antsRegistration (Avants et al., 2008), using brain-extracted versions of both T1w volume and template. Brain tissue segmentation of cerebrospinal fluid, white-matter and grey matter was performed on the brain-extracted T1w using FAST (FSL v. 5.0.9) (Zhang et al., 2001).

For each BOLD run, the following preprocessing was performed: first, a reference volume and its skull-stripped version were generated using a custom methodology of fMRIPrep. A deformation field to correct for susceptibility distortions was estimated based on a field map that was co-registered to the BOLD reference, using a custom workflow of fMRIPrep derived from D. Greve's epidewarp.fs/ script and further improvements of HCP Pipelines (Glasser et al., 2013). Based on the estimated susceptibility distortion, an unwarped BOLD reference was calculated for a more accurate coregistration with the anatomical reference. The BOLD reference was then co-registered to the $\mathrm{T} 1 \mathrm{w}$ reference using bbregister (FreeSurfer). Co-registration was configured with six degrees of freedom. Head-motion parameters with respect to the BOLD reference (transformation matrices, and six 
corresponding rotation and translation parameters) were estimated before any spatiotemporal filtering using mcflirt (FSL v. 5.0.9) (Jenkinson et al., 2002). BOLD runs were slice-time corrected using 3dTshift from AFNI v. 20160207 (Cox and Hyde, 1997). The BOLD time-series (including slicetiming correction when applied) were resampled onto their original, native space by applying a single, composite transform to correct for head-motion and susceptibility distortions. These resampled BOLD time-series will be referred to as preprocessed BOLD in original space, or just preprocessed BOLD. Several confounding time-series were calculated based on the preprocessed BOLD: framewise displacement (FD) was calculated for each functional run, using Nipype's implementation (following the definitions by Power and colleagues (2014)). Additionally, a set of physiological regressors were extracted to allow for component-based noise correction (CompCor) (Behzadi et al., 2007). Principal components were estimated after high-pass filtering the preprocessed BOLD time-series (using a discrete cosine filter with 128s cut-off). A subcortical mask was obtained by heavily eroding the brain mask to ensure it would not include cortical grey matter regions. Six anatomical CompCor (aCompCor) components were then calculated within the intersection of the aforementioned mask and the union of cerebrospinal fluid and white matter masks calculated in T1w space, after their projection to the native space of each functional run (using the inverse BOLD-toT1w transformation). The head-motion estimates calculated in the correction step were also placed within the corresponding confounds file. All resamplings were performed with a single interpolation step by composing all the pertinent transformations (i.e., head-motion transform matrices, susceptibility distortion correction when available, and co-registrations to anatomical and template spaces). Gridded (volumetric) resamplings were performed using antsApplyTransforms (ANTs), configured with Lanczos interpolation to minimize the smoothing effects of other kernels (Lanczos, 1964). Non-gridded (surface) resamplings were performed using mri_vol2surf (FreeSurfer).

\subsubsection{Correlational PPI estimation}

We estimated the first-level whole-brain psychophysiological interaction (cPPI) matrix in each subject's native space. Note that, in contrast with the traditional PPI technique, cPPI results in 
symmetrical, undirected connectivity matrices. We used a region-of-interest (ROI)-based approach, obtaining connectivity terms for $|\mathrm{N}|=416$ ROls corresponding to the cortical Schaeffer parcellation $(|\mathrm{N}|=400)$ (Schaefer et al., 2018) and eight bilateral ROls from the aseg atlas (accumbens, amygdala, caudate, pallidum, putamen, thalamus, hippocampus anterior and posterior) (Fischl et al., 2002). The different conditions ("tasks") of interest were modeled based as events with onsets and durations corresponding to the experimental trial period (i.e., 2 seconds epochs that comprised the entire period of picture presentation). The task regressors were convolved with a double-gamma canonical hemodynamic response function (HRF). Events were assigned to a given condition based on the participant's response during the subsequent memory test namely: Source (subsequent itemsource association [Yes response to Q1 and Q2 and correct response to Q3]), Item (subsequent item memory without memory for the association [correct Yes response to Q1 and either a No response to Q2, or incorrect response to Q3]), Miss memory trials, and trials with no response. BOLD data (average time series) for the 416 ROls were deconvolved into estimates of neural events (Gitelman et al., 2003). Each task time course from the first-level activity GLM design matrix was multiplied separately by the deconvolved neural estimates from the seed region and convolved with a canonical HRF, creating PPI terms. Pairwise (ROI-to-ROI) partial Pearson's correlations were estimated for each participant separately by correlating the ROI-specific source memory PPI terms while controlling for (i) the remaining PPI regressors, (ii) the observed BOLD signal in both regions, and (iii) the original HRF-convolved task regressors. All correlation coefficients were Fishertransformed to $z$ values. Finally, cPPI values were demeaned within-individual to account for nonneural effects in the implicit baseline. The individual source-connectivity matrices were used for higher-level analysis. For illustrative and communication purposes, the ROls were grouped based on 18 networks (subcortical network plus 17 cortical networks as defined by Yeo and colleagues (2011). 


\subsubsection{Longitudinal structural preprocessing}

For the structural longitudinal analysis, we performed cortical reconstruction and volumetric segmentation of the T1w scans using the longitudinal FreeSurfer stream v.6.0 (Reuter et al., 2012) (http://surfer.nmr.mgh.harvard.edu/fswiki). The images were initially processed using the crosssectional stream thoroughly described elsewhere (Dale et al., 1999; Fischl and Dale, 2000; Fischl et al., 1999). The automatized processing pipeline includes removal of nonbrain tissues, Talairach transformation, intensity correction, tissue and volumetric segmentation, cortical surface reconstruction, and cortical parcellation. Next, an unbiased within-subject template volume based on all cross-sectional images was created for each participant, using robust, inverse consistent registration (Reuter et al., 2010). The processing of each time point was then reinitialized with common information from the within-subject template, significantly increasing reliability and statistical power. Before group analysis, cortical hemispheres were brought to fsaverage space and surface smoothing was applied at $12 \mathrm{~mm}$ FWHM. For subcortical structures (i.e., hippocampi) mean bilateral volume for specific structures was used in the analyses.

\subsection{Higher-level analysis}

\subsubsection{Main effects of whole-brain correlational PPI}

We ran four GLM models on the whole-brain connectivity cPPI matrices to assess the mean connectivity and the effects of Age, Performance and AgexPerformance interaction. Sex was used as a covariate of no-interest in all models. The models were built in a step-wise manner, adding complexity in each model. First, we assessed the mean patterns of task-dependent connectivity during memory encoding. Next, we added an age regressor to test for changes in encodingconnectivity with age. The third model tested the relation of performance (as defined by the corrected source memory scores) on encoding connectivity, age controlled. In the fourth model, we tested the Age $\times$ Performance interaction by adding the interaction regressor. This later model was restricted to edges showing a main effect of Performance. All analyses were corrected for multiple comparisons via cluster correction routines from the Network Based Statistics (NBS) toolbox (Zalesky et al., 2010), 
with $p<0.01$ cluster-forming threshold and $p<0.025$ (2 comparisons) cluster significance as determined by permutation testing $(n=5000)$.

\subsubsection{Spatial relationship between connectivity maps and term-based meta-analyses}

To study the relationship between connectivity patterns and cognitive processes, we compared the topology of the main effects of Age, Performance and AgexPerformance interaction with the metaanalytic patterns of activity that were associated with specific cognitive processes.

For connectivity, we estimated the "significance degree" of each ROI in the cPPI graphs; that is the number of connections ("edges") that were significant for a given ROI in a given contrast (Age, Performance, and AgexPerformance interaction). The connectivity output for each contrast was a $|\mathrm{N}|=416$ ROls map representing the degree to which each region was related to Age, Performance, and AgexPerformance interaction effects

The meta-analytic cognitive maps were computed with the NiMARE package (Salo et al., 2018), which uses core functions from Neurosynth (Yarkoni et al., 2011). The software computes metaanalytical maps based on (mostly) activity contrasts in fMRI studies using automated text mining and coordinate extraction tools. We restricted the meta-analytical terms to those that overlapped between the Neurosynth database and the cognitive atlas (Poldrack et al., 2011) $(|N|=123$ terms) thus restricting terms to specific "mental processes" (cognitive and emotional). Coordinate-based multilevel kernel density analysis (MKDA) models were used to model the specificity of the cognitive processes on neuroimaging data (Wager et al., 2009). Specificity refers to the probability of a cognitive term occurring given activation in a specific brain area. We set a term frequency threshold $=0.001$ and a kernel radius $=10 \mathrm{~mm}$. The remaining parameters were left to default. For comparison with "significance degree" from connectivity, the resulting meta-analytical maps were parcellated into $|\mathrm{N}|=416$ ROls using a volumetric parcellation . 
The relationship between the "significant degree" and the meta-analytical cognitive maps was assessed using Pearson's correlations. Permutation-based significance testing $(p \leq 0.01)$ was performed with the BrainSMASH package (Brain Surrogate Maps with Autocorrelated Spatial Heterogeneity) (Burt et al., 2020). BrainSMASH enables statistical testing of spatially correlated brain maps by simulating surrogate brain maps with a spatial autocorrelation that matches the target map; here the meta-analytical cognitive maps (Viladomat et al., 2014). Surrogate maps $(n=5000)$ were generated based on a Euclidean distance matrix of the center-of-gravity ROI coordinates. A null distribution was then defined by correlating the surrogate and the "significant degree" maps.

\subsubsection{Relationship between connectivity patterns in older-age and brain structural decline}

We studied the relationship between encoding connectivity and brain atrophy and cortical thinning in a subsample of older individuals with retrospective longitudinal data ( $n=81$, age $>50$ years). The longitudinal data spanned back on average $8.1(S D=0.93)$ years; the timing of the last observation overlapped with the timing of the encoding task. We focused on the clusters that were associated with memory performance with increasing age. We used a summarized metric that consisted of mean encoding connectivity from the clusters identified in the AgexPerformance interaction models (see above for more details). Hereafter, we refer to those metrics as memory-positive and memorynegative in older age, as the resulting clusters were associated either with higher and lower memory performance with higher age, respectively. We tested whether these patterns of connectivity were associated with whole-brain cortical thinning using Spatiotemporal Linear Mixed Effect (LME) Modelling as implemented in Freesurfer (Bernal-Rusiel et al., 2013). LME models were run as a function of Time (years from the experimental task), Connectivity and the Connectivity $\times$ Time interaction. Sex, Estimated Intracranial Volume (elCV), and Baseline Age (last measurement) were introduced as covariates of no-interest and subject identifiers as random intercepts (Bernal-Rusiel et al., 2013). Statistical significance was tested at each cortical vertex and the resulting maps were corrected for multiple comparisons using False Discovery Rate ( $\mathrm{pFDR}<0.01$ ). Finally, we investigated whether these patterns of connectivity were associated with decreased hippocampus volume, using the same model described above. 


\subsubsection{Relationship between connectivity patterns in older age and cognitive decline}

We studied the relationship between encoding connectivity and decline in memory function and general cognition in a subsample of older individuals with retrospective longitudinal cognitive data. The longitudinal data spanned back on average 7.42 years $(S D=1.94)$; the last observation corresponded in time with the current experimental task. We selected total learning score from the California Verbal Learning Test (CVLT), and the Vocabulary and Matrix Reasoning test from the WASI-II battery as proxies for memory function, crystalized and fluid intelligence. To explore whether connectivity in the memory-positive and memory-negative in older age clusters were associated with cognitive decline over time we ran LME analyses as detailed above with the cognitive measures fitted as a function of Time (years from the experimental task), Connectivity and the Connectivity $\times$ Time interaction $(\mathrm{pFDR}<0.01)$. Sex, and Baseline Age were introduced as covariates of no-interest and subject identifiers as random intercepts.

\section{Results}

\subsection{Behavioral results}

Memory performance in the fMRI task showed a non-linear negative relationship to age, accelerating in the sixth decade of life $(F=52.26[p<0.001])$. The different cognitive measures were related to age (all p's < .001), controlling for sex; higher age was related to lower memory and visuospatial reasoning and higher vocabulary performance. See Fig. 2 and Supplementary Table 2 for additional information. 


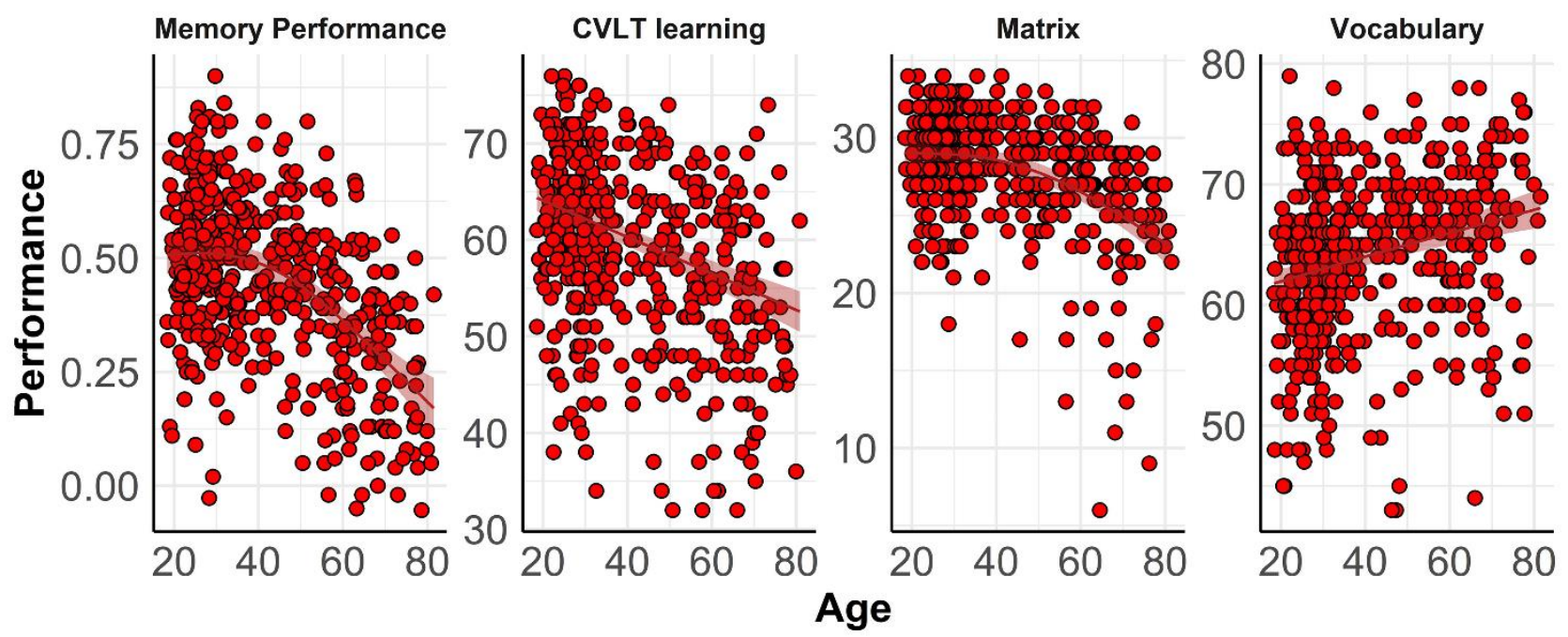

Fig.2 Trajectories of cognition throughout the adult lifespan. Cognitive performance was fitted by age using Generalized Additive Models (GAMs), controlled for sex. Memory performance = corrected source memory score from the experimental task; CVLT learning = words learned and recalled across the five CVLT learning trials; Vocabulary and Matrix = WAIS-IV vocabulary and matrices reasoning raw scores. Ribbons represent $95 \%$ confidence intervals.

\subsection{Whole-brain encoding connectivity}

In the main analyses, we assessed the effect of the mean task connectivity patterns during memory encoding and their association with Age, Performance (corrected source memory score from the experimental task), and the AgexPerformance interaction.

\subsubsection{Mean encoding connectivity}

Mean across-participants connectivity during encoding (Fig. 3A) was characterized by high intranetwork connectivity values and high internetwork connectivity between default-mode subnetworks and somatomotor networks. Low internetwork connectivity of salience, control, and limbic networks with subcortical, visual, somatomotor, and dorsal attention networks was seen.

\subsubsection{Age effects}

Pairwise connectivity between regions involved in higher cognitive functions and unimodal and attentional regions increased with higher age. Specifically, with age connectivity was higher between control, limbic, and default-mode subnetworks with somatomotor, visual, subcortical regions, and 
the dorsal attentional stream. Conversely, intranetwork connectivity was lower in older adults. See

Fig. 3B. As resulted from the Mantel test, we found an inverse relationship $(r=-0.19, p<0.001$ from Mantel test $[\mathrm{n}=10000$ permutations]) between the matrices of mean encoding connectivity and matrices of age effects, suggesting a likely "dedifferentiation" of the connectivity patterns with higher age. The inclusion of Performance in the model did not qualitatively affect the results. 
bioRxiv preprint doi: https://doi org/10.1101/2021.08 10 455779; this version posted August 10, 2021. The copyright holder for this preprint (which was not certified by peer review) is the author/funder, who has granted bioRxiv a license to display the preprint in perpetuity. It is made available under aCC-BY-ND 4.0 International license.

\section{A) Mean Connectivity}

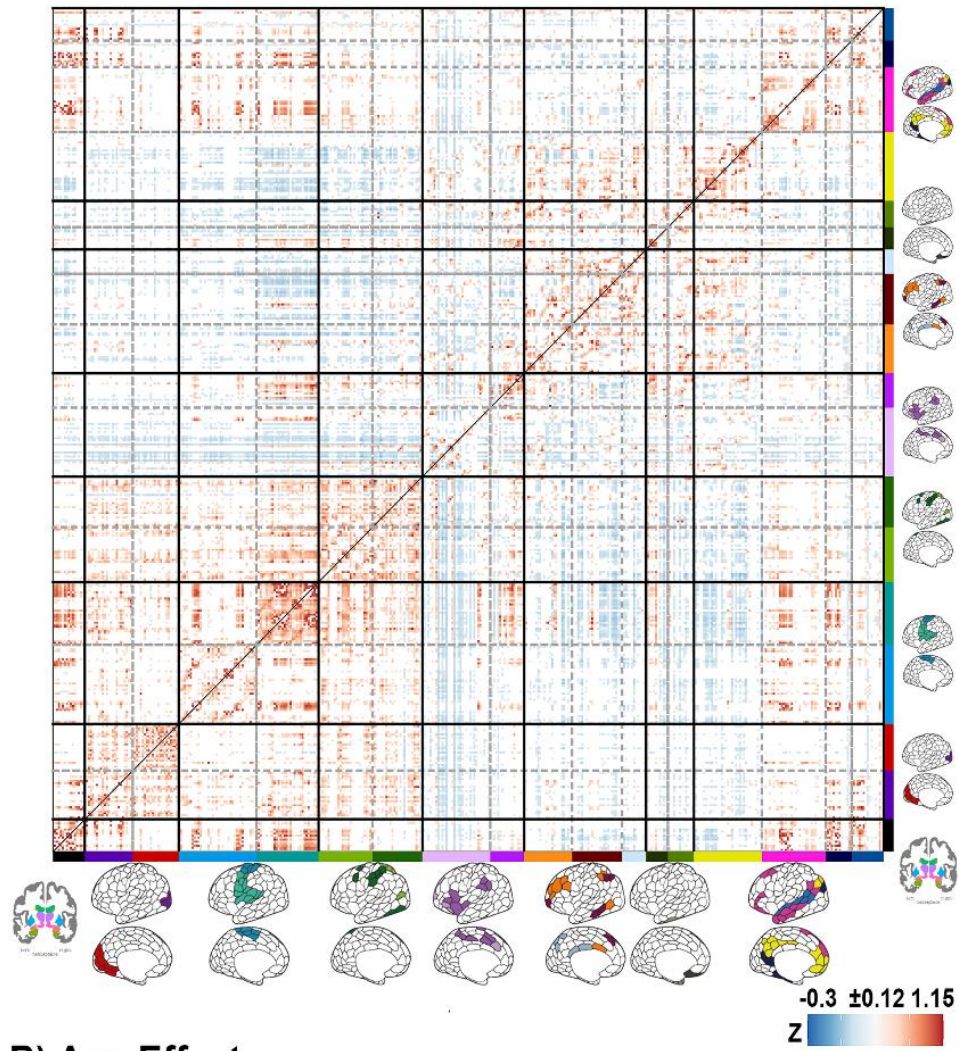

B) Age Effect

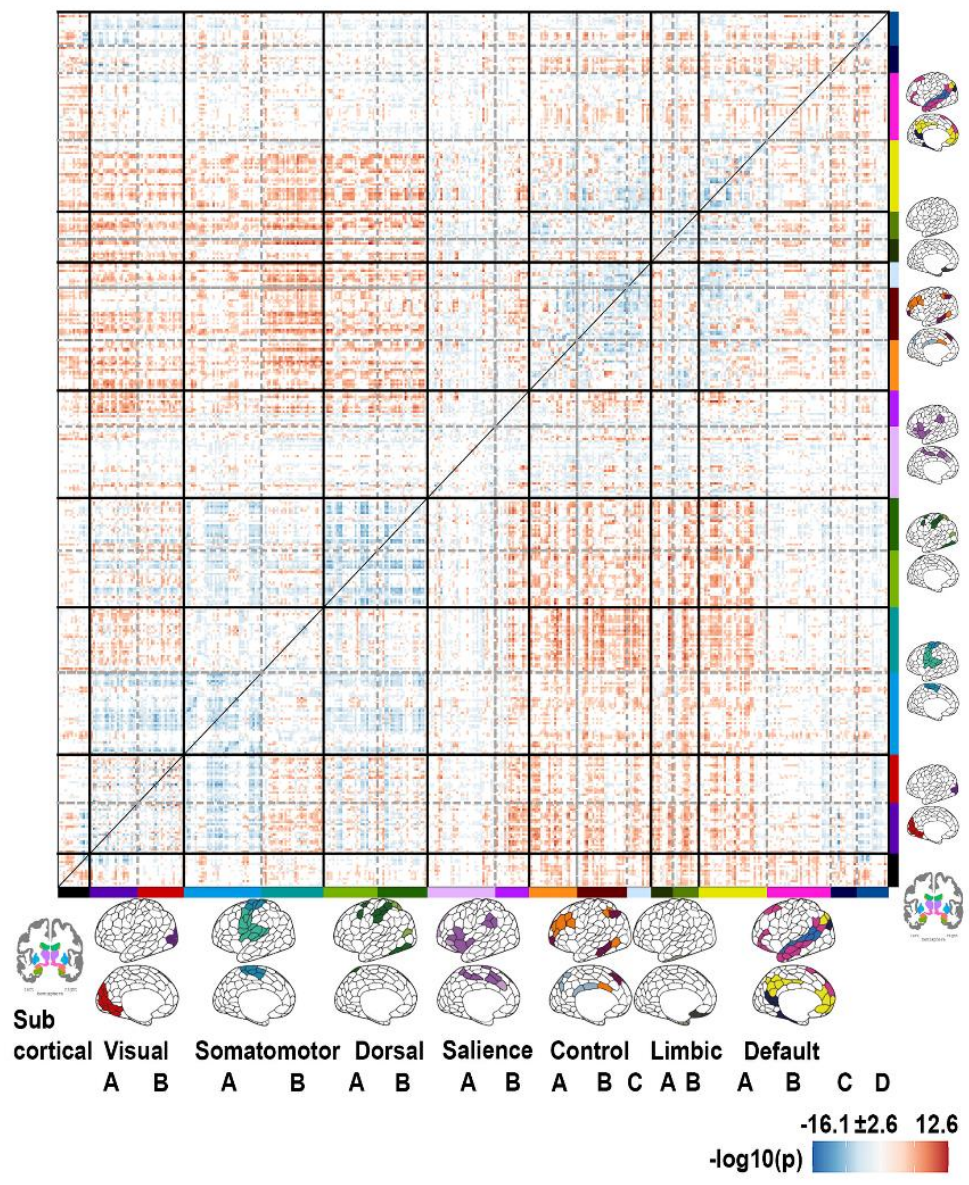


Fig.3 P-values matrices for mean encoding connectivity and age effects. ROls were grouped based on the Yeo-17 atlas (Yeo et al., 2011) and a subcortical network. Default D = TemporoParietal Network. Red represents higher connectivity values and positive age effects, while blue represents lower connectivity values and negative age effects. For the mean effect, only connections $\geq 1 \mathrm{SD}$ of the mean are displayed. For the age effects, only connections in FWEcorrected significant clusters are displayed $(p<0.01)$.

\subsubsection{Performance effects}

Better memory performance (positive performance) in the task was associated with higher connectivity independently of age within posterior parietal and frontal regions, namely the superior parietal lobule, auditory and somatomotor areas, the frontal operculum and medial prefrontal regions (age, sex controlled; Fig. 4B). Conversely, poorer memory performance (negative performance) was associated with higher connectivity within posterior lateral default-mode network regions, medial default-mode network regions, dorsal prefrontal areas, lateral prefrontal areas, and the temporal pole (Fig. 4C). 
bioRxiv preprint doi: $\mathrm{https}$ ///doi.org/10.1101/2021.08.10.455779; this version posted August 10, 2021. The copyright holder for this preprint (which was not certified by peer review) is the author/funder, who has granted bioRxiv a license to display the preprint in perpetuity. It is made available under aCC-BY-ND 4.0 International license.

\section{A) Performance Effect}

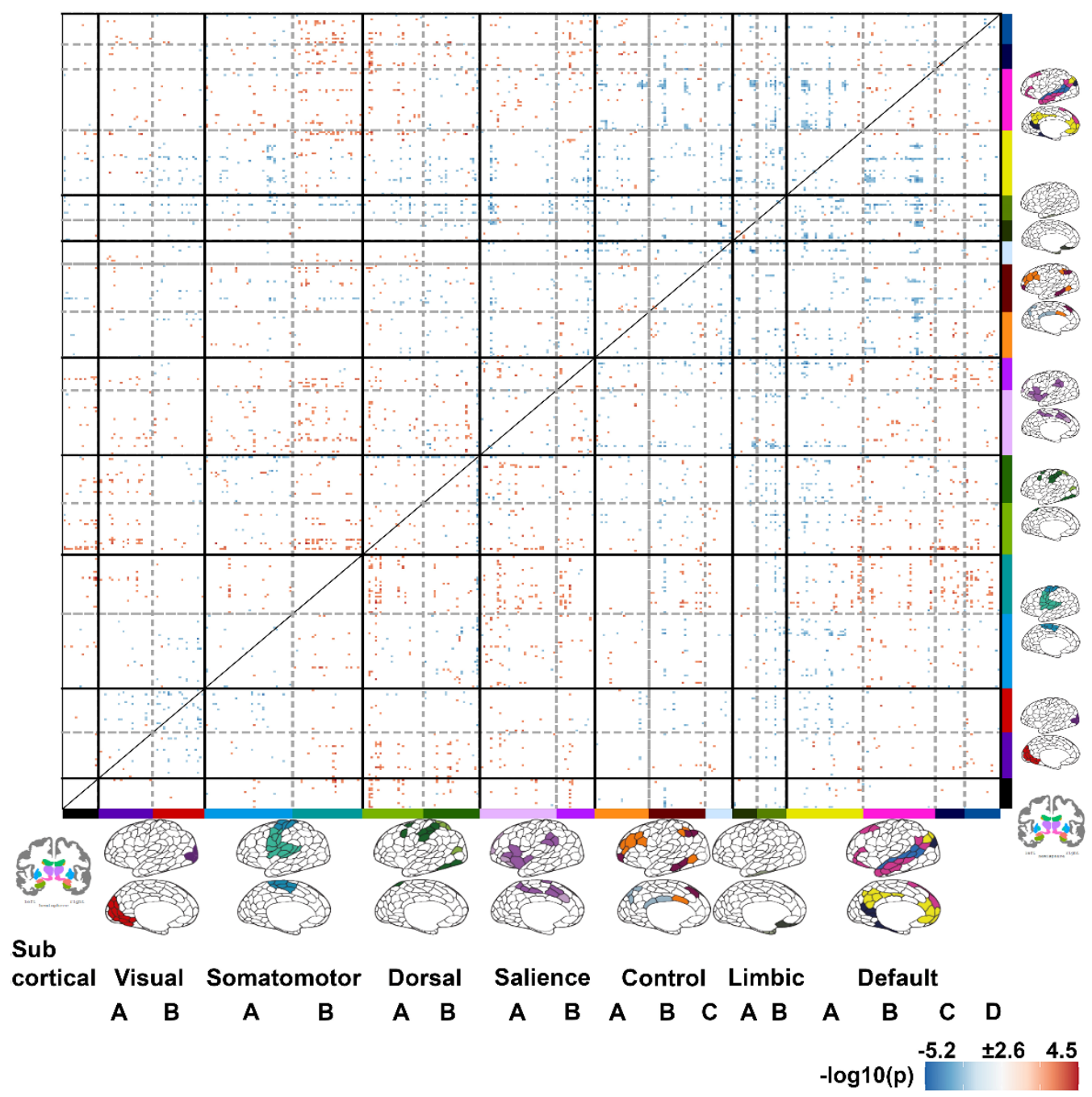

\section{B) Positive Performance}

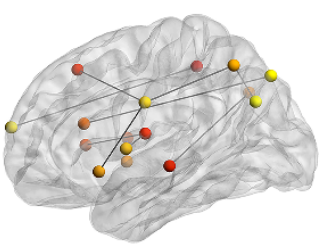

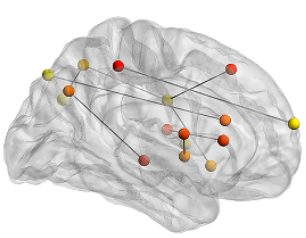

\section{C) Negative Performance}

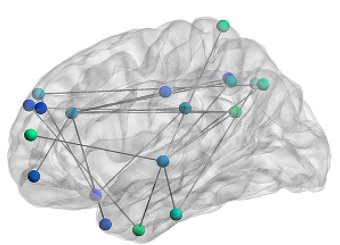

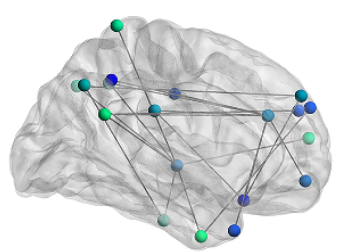

Fig.4 Performance effects. A P-values matrix for performance effects. Performance was defined as (corrected) source memory performance in the experimental task. ROls were grouped based on the Yeo-17 atlas (Yeo et al., 2011) and a subcortical network. Default $D=$ TemporoParietal Network. Red represents higher connectivity values and positive performance effects and vice versa for the blue scale. For the performance effects, only connections within FWEcorrected significant clusters are displayed $(p<0.01)$. B-C Top $5 \%$ of significant nodes shown overlaid to 3D-brain BrainNet Viewer (Xia et al., 2013 http://www.nitrc.org/projects/bnv/). Nodes are filled with red to yellow scales from lower to higher connections (and blue to turquoise from lower to higher connections) that indicate the number of connections ("significance degree"). Only edges between drawn nodes are displayed. 
bioRxiv preprint doi: https://doi.org/10.1101/2021.08.10.455779; this version posted August 10, 2021. The copyright holder for this preprint (which was not certified by peer review) is the author/funder, who has granted bioRxiv a license to display the preprint in perpetuity. It is made available under aCC-BY-ND 4.0 International license.

\subsubsection{AgexPerformance interaction effects}

Next, we tested whether there were AgexPerformance interaction effects within the regions showing a main effect of performance. We found two significant (FWE controlled) clusters showing positive and negative AgexPerformance interactions, respectively. See Fig. $\mathbf{5}$ for a visual illustration. The first cluster (memory-positive in older age) included connectivity between medial temporal and posterior parietal regions, including the retrosplenial cortex, the inferior and superior parietal lobules, and regions in the medial temporal lobe. Higher connectivity between these regions was associated with better performance with higher age (Fig. 5B). The second cluster (memory-negative in older age) corresponded to connections between frontal, parietal, and visual regions. Increased connectivity between these regions was associated with lower performance in older participants (Fig. $5 C)$.

\section{A) Slope AgexPerformance Effect}

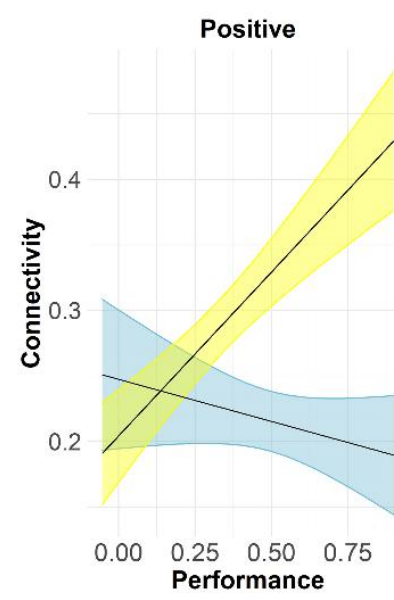

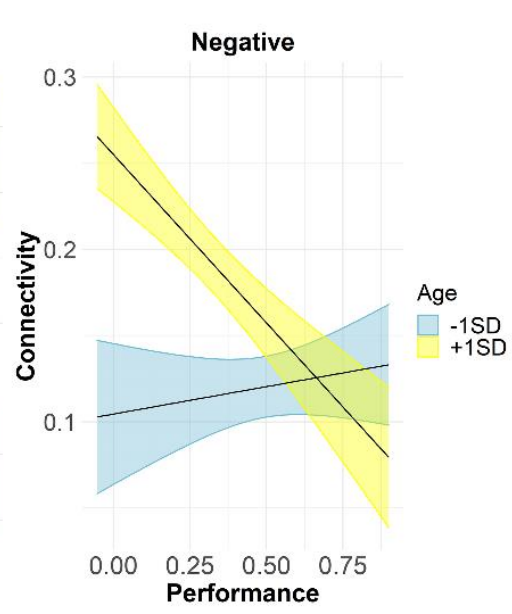

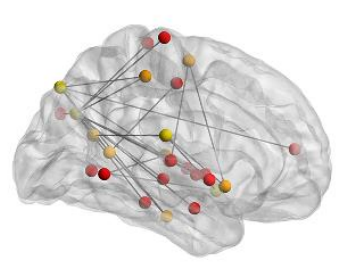

C) Memory-negative in older age
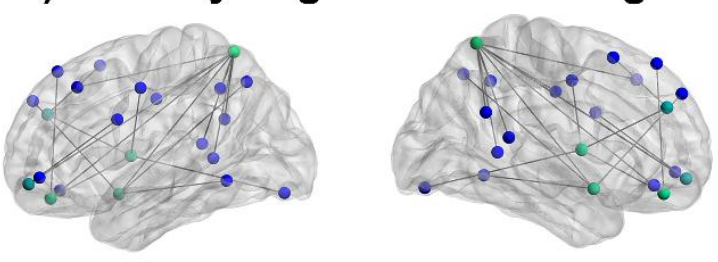

Fig.5 AgexPerformance interaction effects. A Relationship between performance and task-dependent connectivity across age. For illustrative purpose, the effects of performance were predicted at two levels ( \pm 1 SD Age; mean Age = 41.65 [SD $=17.18$ ] years). Note though that Age was introduced as a continuous regressor in the model. Ribbons represent $95 \%$ confidence intervals. B-C Top 5\% of significant nodes shown overlaid to 3D-brain BrainNet Viewer (Xia et al., 2013 http://www.nitrc.org/projects/bnv/). Nodes are filled with red to yellow scales from lower to higher connections (and blue to turquoise from lower to higher connections) that indicate the number of connections ("significance degree"). Only edges between drawn nodes are displayed.

\subsection{Spatial relationship between connectivity maps and term-based meta-analyses}

Next, we tested the spatial relationship between the encoding connectivity patterns and the metaanalytic activity maps associated with specific cognitive processes ( $p \leq 0.01$ using a permutationbased approach). We used a "significance degree" (number of significant connections for a given 
ROI in a given contrast) and specificity metrics for connectivity patterns and cognitive maps, respectively. This was done for all connectivity effects of interest (Age, Performance, and Age $\times$ Performance interaction). See Fig. 6 and Supplementary Table 3 for the full results. The connectivity patterns where greater connectivity was related to higher age (older age) overlapped significantly with the patterns characterizing retrieval, recall, and encoding processes. Conversely, the connectivity patterns where greater connectivity was associated with younger age overlapped with maps related to imagery, spatial attention, and movement activity. The connectivity patterns where greater connectivity was associated with better memory performance overlapped with multisensory, integration and speech production areas. Connectivity patterns where greater connectivity was related to worse performance overlapped with maps associated with salience, emotion, and belief. The spatial patterns of connectivity associated with positive AgexPerformance interaction (memory-positive in older-age) overlapped with the activity patterns associated with mental imagery. No terms were associated with negative AgexPerformance interaction (memorynegative in older age). These results inform us on cognitive processes that may be related to successful memory performance in successful aging, that is, integrative and multisensory strategies and mental imagery. 


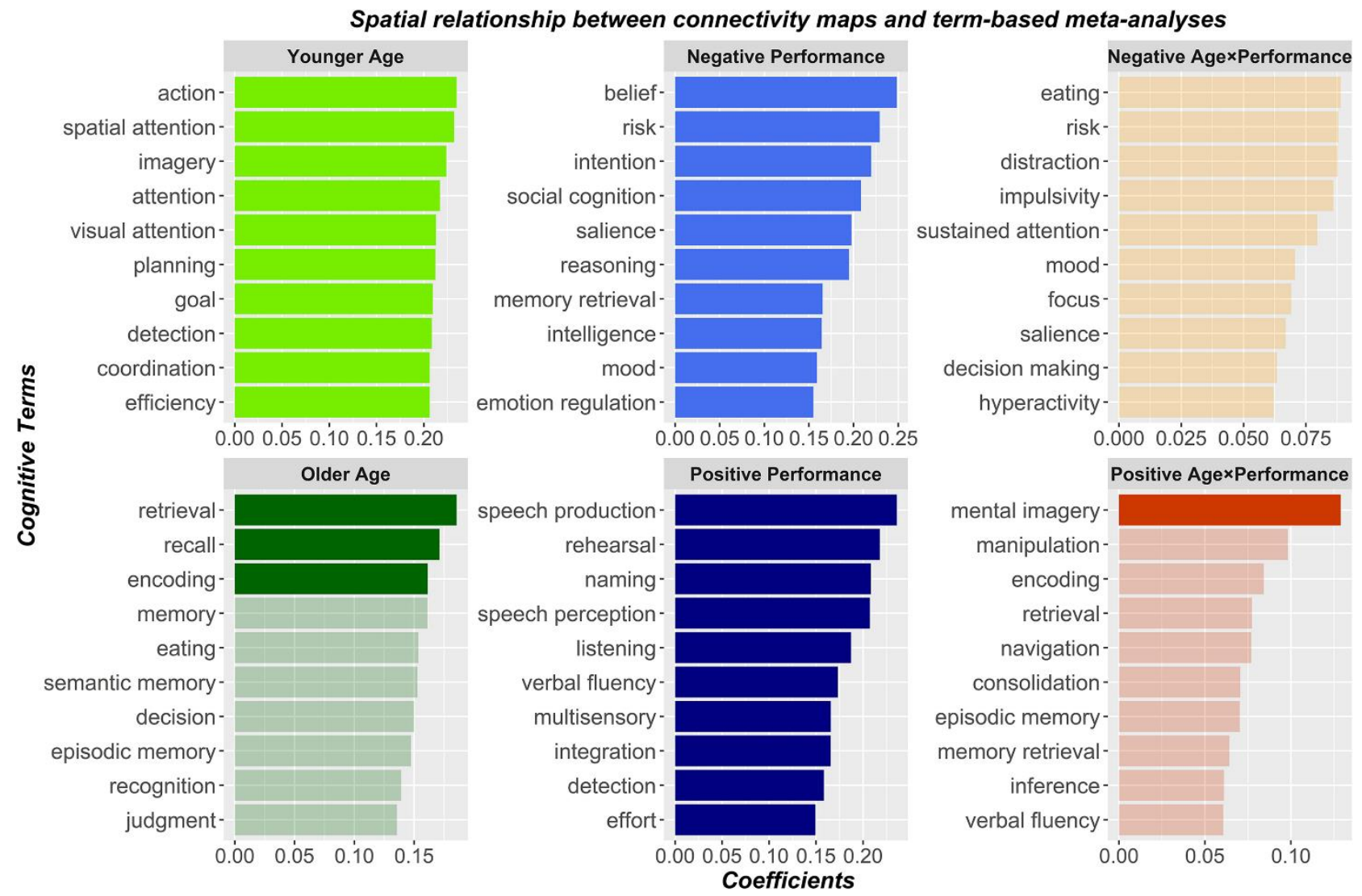

Fig.6 Spatial relationship between connectivity maps and meta-analytic patterns associated with specific cognitive processes. We displayed the top cognitive terms associated with each contrast. Opaque colors reflects terms that survived the significance threshold $(p \leq 0.01)$ as determined by a permutation approach using BrainSMASH (Burt et al., 2020). X-axis represents the empirical Pearson's correlation ( $r$ ), note that different ranges are depicted for each contrast.

\subsection{Relationship between connectivity patterns in older-age and brain structural decline}

We assessed the relationship between brain atrophy and cortical thinning and connectivity patterns in older age to investigate whether age-related connectivity changes reflected maintenance or compensatory responses. This analysis was performed in a subsample of older participants $(n=81$, age $>50$ ) with retrospective longitudinal neuroimaging data (see Supplementary Table 1). A Linear Mixed Effects analysis (controlled for Sex, eICV, and Baseline Age) revealed that the cluster related to higher memory performance in older age (memory-positive in older age) was associated with less hippocampal volume decrease over time $(F=44.2, p<0.001 ;$ Fig.7A $)$. In contrast, the cluster associated with lower memory performance in older age (memory-negative in older age) was related to a steeper volumetric decline of hippocampi $(F=-65.71, p<0.001 ;$ Fig.7B $)$ and cortical thickness decline over time. Indeed, the cortical analysis showed a positive association between connectivity 
in this memory-negative in older age cluster and cortical thinning in two small clusters encompassing

(1) the left precentral and (2) the anterior fusiform and the entorhinal cortices ( $p F D R<0.01$, Fig.7C).

Overall, the results supported the hypothesis that the patterns of connectivity associated with higher and lower performance in older age were related to structural maintenance versus decline of brain regions involved in memory processes.

\section{Memory patterns of connectivity in older age and brain structural decline over time}

A) Memory-positive

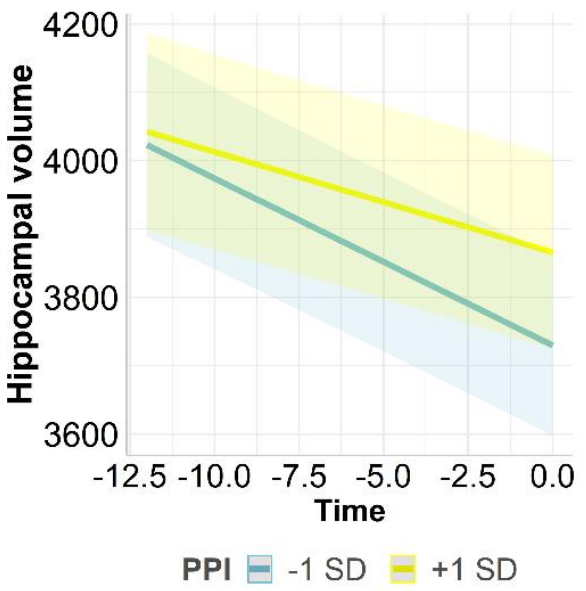

B) Memory-negative

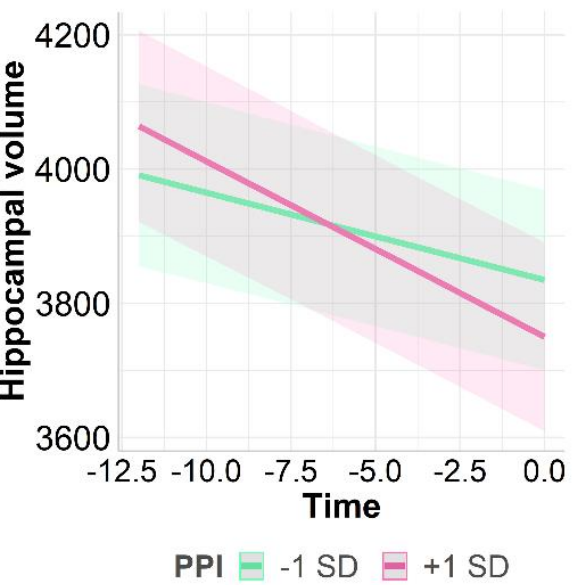

C) Memory-negative in older age and cortical thinning over time

Lat

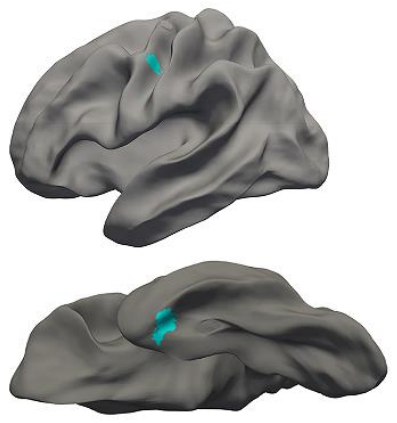

$-5.00-1.671 .675 .00$

Fig.7 Relationship between memory pattern of connectivity in older age and decline of brain structures over time. A-B Relationship between memory patterns of connectivity in older and longitudinal structural hippocampal volume. A The yellow line represents higher memory performance in memory-positive cluster. B The pink line represents lower memory performance in memory-negative cluster. Results are significant at $\mathrm{pFDR}<0.01$. C Whole-brain cortical thickness decline and memory-negative in older age. Only regions showing significant thinning over time are shown. Left hemisphere. Maps are corrected for pFDR $<0.01$. In the colorbar $-\log 10(p)$ values are displayed, red represents higher values, blue lower values.

\subsection{Relationship between connectivity patterns in older age and cognitive decline}

We assessed the relationship between memory patterns of connectivity in older age and cognitive decline using retrospective cognitive data (age > 50). See Supplementary Table 1 for details. LME models (controlled for Sex and Baseline Age) revealed a relationship between connectivity patterns associated with higher memory performance in older age (memory-positive in older age) and less decline in CVLT learning scores. However, the association did not survive multiple comparison corrections $(\mathrm{pFDR}=0.07)$. 


\section{Discussion}

We estimated whole-brain functional connectivity during episodic memory encoding, specifically focusing on age-related differences in connectivity and how they were associated with memory performance across the lifespan. In higher age, we found lower intranetwork and higher internetwork connectivity between regions involved in higher cognitive functions and the dorsal attention stream, sensorimotor and subcortical regions during encoding. Successful memory performance in higher age overlapped with networks involved in mental imagery. Among older adults, greater hippocampal and cortical atrophy was related to less favorable connectivity changes, reflecting maintenance processes over time.

The age effects on encoding connectivity are partially in agreement with previous rs-fMRI and taskfMRI studies, suggesting that some of the functional age-differences are task-independent. For example, several resting-state studies have found reduced intranetwork and increased internetwork connectivity, indicating that brain networks become less specialized during aging (Betzel et al., 2014; Geerligs et al., 2015). Moreover, we found higher age-related connectivity between control and dorsal attention networks, which has been reported previously in a fMRI study using a different task (Grady et al., 2016). This might be interpreted as an over-recruitment of cognitive control processes due to the cognitive demands of the task. Likewise, higher connectivity between inversely engaged networks, such as the control and the dorsal attention respectively with the default-mode, has been described in several tasks in aging (Spreng et al., 2016; Spreng and Turner, 2019). These patterns may reflect age-related features during memory tasks such as lower flexibility in shifting from external and internal attention and semantization of cognition as older individuals might rely more on acquired knowledge. Although speculative, this interpretation receives further support from our results as the connectivity patterns associated with older age mapped unto cognitive processes such as retrieval and recall, suggesting that older participants might rely more on already acquired knowledge and schematic information during the encoding task. However, our results also reveal task-specific 
changes in connectivity. Compared to fMRI acquired during resting-state and other cognitive domains, we also identified the spatial correspondence between the connectivity patterns and cognitive maps, informing us on the specific cognitive processes possibly involved in the task. Younger people exhibited higher connectivity between areas overlapping with regions known to support cognitive processes relevant for our encoding task such as visual attention, action, and imagery. Some of these strategies may also have been adopted by older participants that performed better, which may be interpreted to be in accordance with the maintenance process framework.

We found that the relationship between connectivity and memory performance differed as a function of age. Older people who performed better showed higher connectivity between medial temporal and posterior parietal regions, including the retrosplenial cortex. As shown in a previous study of older participants (Kaboodvand et al., 2018), episodic memory performance was positively associated with functional connectivity between the retrosplenial cortex and the medial temporal lobe. The retrosplenial cortex is a key mediator in facilitating the communication between medial temporal and other default mode networks regions, leading to memory performance success (Kaboodvand et al., 2018). In our study, the connectivity changes that were related to better performance in older participants overlapped spatially with the maps associated with mental imagery, in which the engagement of the retrosplenial cortex is widely described (Chrastil, 2018). These strategies and functional connectivity changes mimicked those associated with younger age.

The patterns of connectivity associated with successful performance in older participants were related to volumetric maintenance of the hippocampus, critically involved in memory encoding. This is in agreement with the maintenance theory of cognitive aging (Nyberg et al., 2012), as hippocampal decline is a main factor behind memory decline in older age (Gorbach et al., 2017). Conversely, we found no evidence that the pattern of connectivity associated with higher performance in older age reflected a compensatory attempt to overcome structural decline. The widespread over-recruitment of different regions in frontal, parietal, and visual areas was not related to memory performance. 
Indeed, these connectivity changes were associated with lower performance and structural loss in the medial temporal lobe over time. Cognitive decline was associated with structural decline and a maladaptive organization in the functional architecture, whereas successful memory performance in older participants reflected relative structural integrity over time and functional connectivity changes that supported the use of "younger" cognitive strategies such as mental imagery.

\subsection{Limitations and methodological remarks}

fMRI during task performance is suited for investigating brain dynamics associated with specific cognitive processes as they possess experimental control while participants perform a task-ofinterest (Campbell and Schacter, 2017). Despite commonalities across states, the functional brain architecture differs across task contexts (Davis et al., 2017) as brain regions reconfigure their connectivity patterns in a flexible way based on the current demands of the task (Cole et al., 2013). This point is supported in our study, as the connectivity patterns associated with successful memory performance mapped on networks involved in cognitive processes relevant for this encoding task. The main disadvantage of task-fMRI is that differences in the experimental design may hamper generalization (Damoiseaux and Huijbers, 2017). Some of our findings agree with previous rs-fMRI and task-fMRI research and thus likely represent task-invariant features of the aging brain. However, other findings, as highlighted by the spatial correlation between functional connectivity and mental imagery processes, seem more constrained to the specific demands of the task, although they relate to real-life function.

The cPPI framework allowed for a whole-brain undirected (symmetric) assessment of task connectivity. cPPI does not imply inferences of directionality. The cPPI connectivity values reflect correlations between regions during selected task-periods of an fMRI run, controlling for stimulusdriven co-fluctuations and intrinsic functional connectivity between the ROls. When cPPI connectivity values estimated from different task-periods are subtracted, the resulting metric is largely comparable to traditional regression-based PPI approaches. However, when conditions are not 
subtracted - as in the current paper - cPPI is akin to "residualised" task-connectivity and beta series correlation, i.e. the similarity between two regions' trial-to-trial fluctuations in BOLD amplitude during task (Di et al., 2020). The different implications of the metrics are largely omitted in the literature but have consequential implications for the interpretation, that is in this case, a task-state of integrated connectivity, instead of a shift in connectivity driven by the specific task.

The effects of demeaning data within participants are also consequential for the interpretation of our results. This approach minimizes the possibility of non-neural confounds that affect the implicit baseline being the main drivers of connectivity differences across individuals. Some of these confounds are known to be greatly correlated with age (Campbell and Schacter, 2017). However, data demeaning only allowed us to interpret the results in relative terms, and in terms of reorganization. Note that many graph-theoretical studies use thresholded, binarized data and thus face a similar problem. It is however possible that some findings are a side-effect of this step. For example, the patterns of connectivity associated with lower performance in older participants are spatially unstructured and thus might represent unspecific changes in the functional connectome rather than reduced functional connectivity amongst specific regions.

Despite the structural and cognitive retrospective longitudinal data available, the main limitation of this study is the lack of longitudinal task-fMRI, which would have allowed us to assess how the functional architecture of the brain during memory tasks changes over time.

\section{Conclusions}


This study provides novel insights in whole-brain connectivity during encoding and its relation with age, cognitive processes and structural decline in older age using a large sample encompassing the entire adulthood. Connectivity patterns underlying successful memory function in older age spatially mapped onto mental imagery processes and were related to structural brain maintenance over time. These results provide a bridge between the cognitive processes and the biological mechanisms that support memory function maintenance and decline in older age.

\section{Disclosure statements}

The authors have no conflicts of interest to disclose.

\section{Acknowledgements}

This work was supported by the Department of Psychology, University of Oslo (to K.B.W., A.M.F.), the Norwegian Research Council (to K.B.W., A.M.F.) and the project has received funding from the European Research Council's Starting Grant scheme under grant agreements 283634, 725025 (to A.M.F.) and 313440 (to K.B.W.). 


\section{References}

Avants, B.B., Epstein, C.L., Grossman, M., Gee, J.C., 2008. Symmetric diffeomorphic image registration with cross-correlation: evaluating automated labeling of elderly and neurodegenerative brain. Med. Image Anal. 12, 26-41. https://doi.org/10.1016/j.media.2007.06.004

Behzadi, Y., Restom, K., Liau, J., Liu, T.T., 2007. A component based noise correction method (CompCor) for BOLD and perfusion based fMRI. Neurolmage 37, 90-101.

https://doi.org/10.1016/j.neuroimage.2007.04.042

Bernal-Rusiel, J.L., Greve, D.N., Reuter, M., Fischl, B., Sabuncu, M.R., 2013. Statistical analysis of longitudinal neuroimage data with Linear Mixed Effects models. Neurolmage 66, 249-260. https://doi.org/10.1016/j.neuroimage.2012.10.065

Betzel, R.F., Byrge, L., He, Y., Goñi, J., Zuo, X.-N., Sporns, O., 2014. Changes in structural and functional connectivity among resting-state networks across the human lifespan. Neurolmage 102 Pt 2, 345-357. https://doi.org/10.1016/j.neuroimage.2014.07.067

Burt, J.B., Helmer, M., Shinn, M., Anticevic, A., Murray, J.D., 2020. Generative modeling of brain maps with spatial autocorrelation. Neurolmage 220, 117038.

https://doi.org/10.1016/j.neuroimage.2020.117038

Cabeza, R., Albert, M., Belleville, S., Craik, F.I.M., Duarte, A., Grady, C.L., Lindenberger, U., Nyberg, L., Park, D.C., Reuter-Lorenz, P.A., Rugg, M.D., Steffener, J., Rajah, M.N., 2018. Maintenance, reserve and compensation: the cognitive neuroscience of healthy ageing. Nat. Rev. Neurosci. 19, 701-710. https://doi.org/10.1038/s41583-018-0068-2

Campbell, K.L., Schacter, D.L., 2017. Ageing and the resting state: is cognition obsolete? Lang. Cogn. Neurosci. 32, 661-668. https://doi.org/10.1080/23273798.2016.1227858

Chrastil, E.R., 2018. Heterogeneity in human retrosplenial cortex: A review of function and connectivity. Behav. Neurosci. 132, 317-338. https://doi.org/10.1037/bne0000261

Cole, M.W., Reynolds, J.R., Power, J.D., Repovs, G., Anticevic, A., Braver, T.S., 2013. Multi-task connectivity reveals flexible hubs for adaptive task control. Nat. Neurosci. 16, 1348-1355. https://doi.org/10.1038/nn.3470

Cox, R.W., Hyde, J.S., 1997. Software tools for analysis and visualization of fMRI data. NMR Biomed. 10, 171-178. https://doi.org/10.1002/(sici)1099-1492(199706/08)10:4/5<171::aidnbm453>3.0.co;2-I

Dale, A.M., Fischl, B., Sereno, M.I., 1999. Cortical surface-based analysis. I. Segmentation and surface reconstruction. Neurolmage 9, 179-194. https://doi.org/10.1006/nimg.1998.0395

Damoiseaux, J.S., Huijbers, W., 2017. The complementary value of task-evoked and resting-state functional imaging: a commentary on Campbell and Schacter (2016). Lang. Cogn. Neurosci. 32, 678-680. https://doi.org/10.1080/23273798.2016.1248452

Davis, S.W., Stanley, M.L., Moscovitch, M., Cabeza, R., 2017. Resting-state networks do not determine cognitive function networks: a commentary on Campbell and Schacter (2016). Lang. Cogn. Neurosci. 32, 669-673. https://doi.org/10.1080/23273798.2016.1252847

Delis, D.C., Kramer, J.H., Kaplan, E., Ober, B.A., 2000. Manual for the California verbal learning test,(CVLT-II). San Antonio TX Psychol. Corp. 
Deng, L., Stanley, M.L., Monge, Z.A., Wing, E.A., Geib, B.R., Davis, S.W., Cabeza, R., 2021. AgeRelated Compensatory Reconfiguration of PFC Connections during Episodic Memory Retrieval. Cereb. Cortex N. Y. N 1991 31, 717-730. https://doi.org/10.1093/cercor/bhaa192

Di, X., Zhang, Z., Biswal, B.B., 2020. Understanding psychophysiological interaction and its relations to beta series correlation. Brain Imaging Behav. https://doi.org/10.1007/s11682-02000304-8

Esteban, O., Markiewicz, C.J., Blair, R.W., Moodie, C.A., Isik, A.I., Erramuzpe, A., Kent, J.D., Goncalves, M., DuPre, E., Snyder, M., Oya, H., Ghosh, S.S., Wright, J., Durnez, J., Poldrack, R.A., Gorgolewski, K.J., 2019. fMRIPrep: a robust preprocessing pipeline for functional MRI. Nat. Methods 16, 111-116. https://doi.org/10.1038/s41592-018-0235-4

Fischl, B., Dale, A.M., 2000. Measuring the thickness of the human cerebral cortex from magnetic resonance images. Proc. Natl. Acad. Sci. U. S. A. 97, 11050-11055. https://doi.org/10.1073/pnas.200033797

Fischl, B., Salat, D.H., Busa, E., Albert, M., Dieterich, M., Haselgrove, C., van der Kouwe, A., Killiany, R., Kennedy, D., Klaveness, S., Montillo, A., Makris, N., Rosen, B., Dale, A.M., 2002. Whole Brain Segmentation. Neuron 33, 341-355. https://doi.org/10.1016/S0896-6273(02)00569-X

Fischl, B., Sereno, M.I., Dale, A.M., 1999. Cortical surface-based analysis. II: Inflation, flattening, and a surface-based coordinate system. Neurolmage 9, 195-207. https://doi.org/10.1006/nimg.1998.0396

Fjell, A.M., Sneve, M.H., Grydeland, H., Storsve, A.B., de Lange, A.-M.G., Amlien, I.K., Røgeberg, O.J., Walhovd, K.B., 2015. Functional connectivity change across multiple cortical networks relates to episodic memory changes in aging. Neurobiol. Aging 36, 3255-3268.

https://doi.org/10.1016/j.neurobiolaging.2015.08.020

Folstein, M.F., Folstein, S.E., McHugh, P.R., 1975. "Mini-mental state". A practical method for grading the cognitive state of patients for the clinician. J. Psychiatr. Res. 12, 189-198. https://doi.org/10.1016/0022-3956(75)90026-6

Fonov, V., Evans, A., McKinstry, R., Almli, C., Collins, D., 2009. Unbiased nonlinear average ageappropriate brain templates from birth to adulthood. Neurolmage 47, S102.

https://doi.org/10.1016/S1053-8119(09)70884-5

Fornito, A., Harrison, B.J., Zalesky, A., Simons, J.S., 2012. Competitive and cooperative dynamics of large-scale brain functional networks supporting recollection. Proc. Natl. Acad. Sci. 109, 1278812793. https://doi.org/10.1073/pnas.1204185109

Geerligs, L., Renken, R.J., Saliasi, E., Maurits, N.M., Lorist, M.M., 2015. A Brain-Wide Study of Age-Related Changes in Functional Connectivity. Cereb. Cortex 25, 1987-1999. https://doi.org/10.1093/cercor/bhu012

Gitelman, D.R., Penny, W.D., Ashburner, J., Friston, K.J., 2003. Modeling regional and psychophysiologic interactions in $\mathrm{fMRI}$ : the importance of hemodynamic deconvolution. Neurolmage 19, 200-207. https://doi.org/10.1016/S1053-8119(03)00058-2

Glasser, M.F., Sotiropoulos, S.N., Wilson, J.A., Coalson, T.S., Fischl, B., Andersson, J.L., Xu, J., Jbabdi, S., Webster, M., Polimeni, J.R., Van Essen, D.C., Jenkinson, M., WU-Minn HCP Consortium, 2013. The minimal preprocessing pipelines for the Human Connectome Project. Neurolmage 80, 105-124. https://doi.org/10.1016/j.neuroimage.2013.04.127 
Gorbach, T., Pudas, S., Lundquist, A., Orädd, G., Josefsson, M., Salami, A., de Luna, X., Nyberg, L., 2017. Longitudinal association between hippocampus atrophy and episodic-memory decline. Neurobiol. Aging 51, 167-176. https://doi.org/10.1016/j.neurobiolaging.2016.12.002

Gorgolewski, K.J., Esteban, O., Markiewicz, C.J., Ziegler, E., Ellis, D.G., Jarecka, D., Notter, M.P., Johnson, H., Burns, C., Manhães-Savio, A., 2018. nipy/nipype: 1.1.6. Zenodo. https://doi.org/10.5281/zenodo.1560596

Grady, C., 2012. The cognitive neuroscience of ageing. Nat. Rev. Neurosci. 13, 491-505. https://doi.org/10.1038/nrn3256

Grady, C., Sarraf, S., Saverino, C., Campbell, K., 2016. Age differences in the functional interactions among the default, frontoparietal control, and dorsal attention networks. Neurobiol. Aging 41, 159-172. https://doi.org/10.1016/j.neurobiolaging.2016.02.020

Grady, C.L., Mclntosh, A.R., Craik, F.I.M., 2003. Age-related differences in the functional connectivity of the hippocampus during memory encoding. Hippocampus $13,572-586$. https://doi.org/10.1002/hipo.10114

Jenkinson, M., Bannister, P., Brady, M., Smith, S., 2002. Improved optimization for the robust and accurate linear registration and motion correction of brain images. Neurolmage 17, 825-841. https://doi.org/10.1016/s1053-8119(02)91132-8

Jovicich, J., Czanner, S., Greve, D., Haley, E., van der Kouwe, A., Gollub, R., Kennedy, D., Schmitt, F., Brown, G., Macfall, J., Fischl, B., Dale, A., 2006. Reliability in multi-site structural MRI studies: effects of gradient non-linearity correction on phantom and human data. Neurolmage 30, 436-443. https://doi.org/10.1016/j.neuroimage.2005.09.046

Kaboodvand, N., Bäckman, L., Nyberg, L., Salami, A., 2018. The retrosplenial cortex: A memory gateway between the cortical default mode network and the medial temporal lobe. Hum. Brain Mapp. 39, 2020-2034. https://doi.org/10.1002/hbm.23983

Keerativittayayut, R., Aoki, R., Sarabi, M.T., Jimura, K., Nakahara, K., 2018. Large-scale network integration in the human brain tracks temporal fluctuations in memory encoding performance. eLife 7, e32696. https://doi.org/10.7554/eLife.32696

Klein, A., Ghosh, S.S., Bao, F.S., Giard, J., Häme, Y., Stavsky, E., Lee, N., Rossa, B., Reuter, M., Chaibub Neto, E., Keshavan, A., 2017. Mindboggling morphometry of human brains. PLoS Comput. Biol. 13, e1005350. https://doi.org/10.1371/journal.pcbi.1005350

Lanczos, C., 1964. Evaluation of Noisy Data. J. Soc. Ind. Appl. Math. Ser. B Numer. Anal. 1, 7685.

Matthäus, F., Schmidt, J.-P., Banerjee, A., Schulze, T.G., Demirakca, T., Diener, C., 2012. Effects of Age on the Structure of Functional Connectivity Networks During Episodic and Working Memory Demand. Brain Connect. 2, 113-124. https://doi.org/10.1089/brain.2012.0077

Nyberg, L., Lövdén, M., Riklund, K., Lindenberger, U., Bäckman, L., 2012. Memory aging and brain maintenance. Trends Cogn. Sci. 16, 292-305. https://doi.org/10.1016/j.tics.2012.04.005

Oh, H., Jagust, W.J., 2013. Frontotemporal Network Connectivity during Memory Encoding Is Increased with Aging and Disrupted by Beta-Amyloid. J. Neurosci. 33, 18425-18437. https://doi.org/10.1523/JNEUROSCI.2775-13.2013

Persson, J., Nyberg, L., Lind, J., Larsson, A., Nilsson, L.-G., Ingvar, M., Buckner, R.L., 2006. Structure-Function Correlates of Cognitive Decline in Aging. Cereb. Cortex 16, 907-915. https://doi.org/10.1093/cercor/bhj036 
Poldrack, R.A., Kittur, A., Kalar, D., Miller, E., Seppa, C., Gil, Y., Parker, D.S., Sabb, F.W., Bilder, R.M., 2011. The Cognitive Atlas: Toward a Knowledge Foundation for Cognitive Neuroscience. Front. Neuroinformatics 5. https://doi.org/10.3389/fninf.2011.00017

Power, J.D., Mitra, A., Laumann, T.O., Snyder, A.Z., Schlaggar, B.L., Petersen, S.E., 2014. Methods to detect, characterize, and remove motion artifact in resting state fMRI. Neurolmage 84, 320-341. https://doi.org/10.1016/j.neuroimage.2013.08.048

Pudas, S., Josefsson, M., Rieckmann, A., Nyberg, L., 2018. Longitudinal Evidence for Increased Functional Response in Frontal Cortex for Older Adults with Hippocampal Atrophy and Memory Decline. Cereb. Cortex 28, 936-948. https://doi.org/10.1093/cercor/bhw418

Reuter, M., Rosas, H.D., Fischl, B., 2010. Highly accurate inverse consistent registration: A robust approach. Neurolmage 53, 1181-1196. https://doi.org/10.1016/j.neuroimage.2010.07.020

Reuter, M., Schmansky, N.J., Rosas, H.D., Fischl, B., 2012. Within-subject template estimation for unbiased longitudinal image analysis. Neurolmage 61, 1402-1418.

https://doi.org/10.1016/j.neuroimage.2012.02.084

Rönnlund, M., Nyberg, L., Bäckman, L., Nilsson, L.-G., 2005. Stability, Growth, and Decline in Adult Life Span Development of Declarative Memory: Cross-Sectional and Longitudinal Data From a Population-Based Study. Psychol. Aging 20, 3-18. https://doi.org/10.1037/0882-7974.20.1.3

Sala-Llonch, R., Bartrés-Faz, D., Junqué, C., 2015. Reorganization of brain networks in aging: a review of functional connectivity studies. Front. Psychol. 6, 663.

https://doi.org/10.3389/fpsyg.2015.00663

Salo, T., Bottenhorn, K.L., Nichols, T.E., Riedel, M.C., Sutherland, M.T., Yarkoni, T., Laird, A.R., 2018. $<$ p $>$ NiMARE: a neuroimaging meta-analysis research environment $</ p>$. F1000Research 7. https://doi.org/10.7490/f1000research.1115905.1

Schaefer, A., Kong, R., Gordon, E.M., Laumann, T.O., Zuo, X.-N., Holmes, A.J., Eickhoff, S.B., Yeo, B.T.T., 2018. Local-Global Parcellation of the Human Cerebral Cortex from Intrinsic Functional Connectivity MRI. Cereb. Cortex 28, 3095-3114. https://doi.org/10.1093/cercor/bhx179

Schedlbauer, A.M., Ekstrom, A.D., 2019. Flexible network community organization during the encoding and retrieval of spatiotemporal episodic memories. Netw. Neurosci. 3, 1070-1093. https://doi.org/10.1162/netn_a_00102

Sled, J.G., Zijdenbos, A.P., Evans, A.C., 1998. A nonparametric method for automatic correction of intensity nonuniformity in MRI data. IEEE Trans. Med. Imaging 17, 87-97.

https://doi.org/10.1109/42.668698

Sneve, M.H., Grydeland, H., Nyberg, L., Bowles, B., Amlien, I.K., Langnes, E., Walhovd, K.B., Fjell, A.M., 2015. Mechanisms Underlying Encoding of Short-Lived Versus Durable Episodic Memories. J. Neurosci. 35, 5202-5212. https://doi.org/10.1523/JNEUROSCI.4434-14.2015

Spreng, R.N., Stevens, W.D., Viviano, J.D., Schacter, D.L., 2016. Attenuated anticorrelation between the default and dorsal attention networks with aging: evidence from task and rest. Neurobiol. Aging 45, 149-160. https://doi.org/10.1016/j.neurobiolaging.2016.05.020

Spreng, R.N., Turner, G.R., 2019. The Shifting Architecture of Cognition and Brain Function in Older Adulthood. Perspect. Psychol. Sci. J. Assoc. Psychol. Sci. 14, 523-542.

https://doi.org/10.1177/1745691619827511 
Tustison, N.J., Avants, B.B., Cook, P.A., Zheng, Y., Egan, A., Yushkevich, P.A., Gee, J.C., 2010. N4ITK: improved N3 bias correction. IEEE Trans. Med. Imaging 29, 1310-1320. https://doi.org/10.1109/TMI.2010.2046908

Vidal-Piñeiro, D., Sneve, M.H., Nyberg, L.H., Mowinckel, A.M., Sederevicius, D., Walhovd, K.B., Fjell, A.M., 2019. Maintained Frontal Activity Underlies High Memory Function Over 8 Years in Aging. Cereb. Cortex 29, 3111-3123. https://doi.org/10.1093/cercor/bhy177

Vidal-Piñeiro, D., Sneve, M.H., Storsve, A.B., Roe, J.M., Walhovd, K.B., Fjell, A.M., 2017. Neural correlates of durable memories across the adult lifespan: brain activity at encoding and retrieval. Neurobiol. Aging 60, 20-33. https://doi.org/10.1016/j.neurobiolaging.2017.08.017

Vidal-Piñeiro, D., Valls-Pedret, C., Fernández-Cabello, S., Arenaza-Urquijo, E.M., Sala-Llonch, R., Solana, E., Bargalló, N., Junqué, C., Ros, E., Bartrés-Faz, D., 2014. Decreased Default Mode Network connectivity correlates with age-associated structural and cognitive changes. Front. Aging Neurosci. 6, 256. https://doi.org/10.3389/fnagi.2014.00256

Viladomat, J., Mazumder, R., McInturff, A., McCauley, D.J., Hastie, T., 2014. Assessing the significance of global and local correlations under spatial autocorrelation: a nonparametric approach. Biometrics 70, 409-418. https://doi.org/10.1111/biom.12139

Wager, T.D., Lindquist, M.A., Nichols, T.E., Kober, H., Van Snellenberg, J.X., 2009. Evaluating the consistency and specificity of neuroimaging data using meta-analysis. Neurolmage 45, S210S221. https://doi.org/10.1016/j.neuroimage.2008.10.061

Wang, L., Li, Y., Metzak, P., He, Y., Woodward, T.S., 2010. Age-related changes in topological patterns of large-scale brain functional networks during memory encoding and recognition. Neurolmage 50, 862-872. https://doi.org/10.1016/j.neuroimage.2010.01.044

Wechsler, T., 1999. Wechsler abbreviated scale of intelligence. San Antonio, TX.

Xia, M., Wang, J., He, Y., 2013. BrainNet Viewer: a network visualization tool for human brain connectomics. PloS One 8, e68910. https://doi.org/10.1371/journal.pone.0068910

Yarkoni, T., Poldrack, R.A., Nichols, T.E., Van Essen, D.C., Wager, T.D., 2011. Large-scale automated synthesis of human functional neuroimaging data. Nat. Methods 8, 665-670. https://doi.org/10.1038/nmeth.1635

Yeo, B.T.T., Krienen, F.M., Sepulcre, J., Sabuncu, M.R., Lashkari, D., Hollinshead, M., Roffman, J.L., Smoller, J.W., Zöllei, L., Polimeni, J.R., Fischl, B., Liu, H., Buckner, R.L., 2011. The organization of the human cerebral cortex estimated by intrinsic functional connectivity. J. Neurophysiol. 106, 1125-1165. https://doi.org/10.1152/jn.00338.2011

Zalesky, A., Fornito, A., Bullmore, E.T., 2010. Network-based statistic: Identifying differences in brain networks. Neurolmage 53, 1197-1207. https://doi.org/10.1016/j.neuroimage.2010.06.041

Zhang, Y., Brady, M., Smith, S., 2001. Segmentation of brain MR images through a hidden Markov random field model and the expectation-maximization algorithm. IEEE Trans. Med. Imaging 20, 45-57. https://doi.org/10.1109/42.906424 